\title{
NUMERICAL MODELING OF NON-EQUILIBRIUM SORPTION PROCESS
}

\author{
I.A. KALIEV, S.T. MUKHAMBETZHANOV, G.S. SABITOVA
}

\begin{abstract}
Filtration in porous media of fluids and gases containing associated with them (dissolved, particulate) solid substances is accompanied by the diffusion of these substances and mass transfer between the liquid (gas) and solid stages. The most common types of mass transfer are sorption and desorption, ion exchange, dissolution and crystallization, mudding, sulfation and suffusion, waxing. We consider the system of equations modeling the process of non-equilibrium sorption. We formulate a difference approximation of the differential problem by an implicit scheme. The solution to the difference problem is constructed by the sweep method. Basing on the numerical results, we can conclude the following: as the relaxation time decreases, the solution to the non-equilibrium problem tends to the solution of the equilibrium problem as the time increases.
\end{abstract}

Keywords: system of equations of non-equilibrium sorption, difference approximation, implicit scheme, sweep method, numerical experiments.

Mathematics Subject Classification: 35Q35, 65M06, 76S05

\section{INTRODUCTION}

Almost all the liquids in the nature are solutions, i.e., they are mixture of two or more substances (components). The filtration in porous media of liquids and gases containing associated (dissolved, suspended) solids is accompanied by the diffusion of these substances and mass transfer between liquid (gas) stage and solid stage. The most common types of mass transfer are sorption and desorption, ion exchange, dissolution and crystallization, mudding, sulfation and suffusion, waxing. Taking into consideration the features of physical and chemical interaction of solutions with reservoir rocks, the problems of equilibrium and non-equilibrium are considered.

\section{Formulation of the PROBlem}

Let $m(x, t)$ be the porosity of a media, $0<m(x, t) \leqslant 1$, the porous space be filled by a liquid and by a precipitated solid phase, $c(x, t)$ be the mass concentration of a certain substance in the liquid phase (per unit of the volume), $s(x, t)$ be the mass concentration of the precipitated solid of this substance (per unit of the volume of the pores).

Under equilibrium conditions when the contact between the solution and the solid is kept for a long time, the relation between the concentrations $c(x, t)$ in the solution and on the sorbate $s(x, t)$ is determined by the sorption isotherm. For small concentrations of the solution, the absorption value is determined by a linear dependence, namely, by Henry's isotherm $s=\Gamma c$, where $\Gamma>0$ is some constant depending on physical and chemical properties of the media (Henry constant).

I.A. Kaliev, S.T. Mukhambetzhanov, G.S. Sabitova, Numerical modeling of the nonEQUILIBRIUM SORPTION PROCESS.

(c) Kaliev I.A.,Mukhambetzhanov S.T., Sabitova G.S. 2016.

Submitted August 18, 2015. 
Equilibrium sorption equation can not always completely characterize the features of sorption and substances exchange in a two-phase system solution-solid. In works [1]-[3] there were proposed mathematical models describing non-equilibrium sorption processes. At that, concentration $s$ of solid stage is related with concentration $c$ in the liquid stage by the equation

$$
\frac{\partial s}{\partial t}=\frac{1}{\tau}(\Gamma c-s)
$$

where positive constant $\tau$ is a character relaxation time, $G$ is Henry constant. Concentration $c$ of a substance in a solution satisfies the equation

$$
m \frac{\partial c}{\partial t}=D \triangle c-v \nabla c-\frac{\partial s}{\partial t}
$$

where $D(x, t)>0$ is the diffusion coefficient, $v(x, t)$ is the filtration rate vector, which are regarded as known functions of the mentioned arguments.

Let $\Omega$ be a bounded domain in a $n$-dimensional space $\mathbb{R}^{n}$ with a sufficiently smooth boundary $S=\partial \Omega, Q_{T}=\Omega \times(0, T), T>0, S_{T}=S \times(0, T)$ be the lateral surface of cylinder $Q_{T}$.

We need to find functions $c(x, t), s(x, t)$ defined in domain $Q_{T}$ satisfying equations (1), (2) in $Q_{T}$, the initial conditions

$$
\begin{aligned}
& c(x, 0)=c_{0}(x), x \in \Omega, \\
& s(x, 0)=s_{0}(x), x \in \Omega,
\end{aligned}
$$

and the boundary condition

$$
c(x, t)=c_{b}(x, t),(x, t) \in S_{T} .
$$

In work [4], there was proved the global unique solvability of multi-dimensional initialboundary value problem (1)-(5) modelling a non-equilibrium sorption process.

\section{Difference approximation of Differential Problem by AN IMPlicit SCheme}

Consider the case of one-dimensional variable $x$. In this case equation (2) is rewritten as

$$
m \frac{\partial c}{\partial t}=D \frac{\partial^{2} c}{\partial x^{2}}-v \frac{\partial c}{\partial x}-\frac{\partial s}{\partial t} .
$$

Let the concentration of the substance in the liquid phase, function $c(x, t)$, satisfies the initial condition

$$
c(x, 0)=c_{0}(x), x \in(0,1)
$$

and the boundary conditions

$$
c(0, t)=c_{b 0}(t), \quad c(1, t)=c_{b 1}(t), \quad t \in[0, T],
$$

while the concentration of the solid stage, function $s(x, t)$, satisfies the initial condition

$$
s(x, 0)=s_{0}(x), x \in(0,1) .
$$

We need to find solution $c(x, t), s(x, t)$ to equations $(1)$ and $(6)$ in the rectangle $(x, t) \in$ $(0,1) \times(0, T)$ satisfying initial conditions $(7),(9)$ and boundary conditions $(8)$.

The main tool of the numerical solving partial differential equations are difference methods. To find an approximate solution of this problem, we consider a rectangular grid of nodes formed by the intersection points of two families of parallel straight lines:

$$
x=i h, \quad i=\overline{0, k}, \quad h=1 / k ; \quad t=j q, \quad j=\overline{0, p}, \quad q=T / p .
$$


For each node $(i, j)$ we denote $c_{i, j}=c(i h, j q), s_{i, j}=s(i h, j q)$ and we write the difference approximation of differential equations (1) and (6)

$$
\begin{aligned}
\frac{s_{i, j+1}-s_{i, j}}{q}=\frac{1}{\tau} & \left(\Gamma c_{i, j}-s_{i, j}\right), \quad i=\overline{0, k}, \quad j=\overline{0, p-1}, \\
m_{i, j} \frac{c_{i, j+1}-c_{i, j}}{q}= & D_{i, j} \frac{c_{i+1, j+1}-2 c_{i, j+1}+c_{i-1, j+1}}{h^{2}} \\
& -v_{i, j} \frac{c_{i+1, j+1}-c_{i-1, j+1}}{2 h}-\frac{s_{i, j+1}-s_{i, j}}{q} .
\end{aligned}
$$

We express $s_{i, j+1}$ from equation (10):

$$
s_{i, j+1}=s_{i, j}\left(1-\frac{q}{\tau}\right)+\frac{q \Gamma}{\tau} c_{i, j} .
$$

By equation (11) we obtain

$$
\begin{aligned}
& \frac{q}{h}\left(\frac{v_{i, j}}{2}-\frac{D_{i, j}}{h}\right) c_{i+1, j+1}+\left(m_{i, j}+\frac{2 D_{i, j} q}{h^{2}}\right) c_{i, j+1} \\
& \quad-\frac{q}{h}\left(\frac{D_{i, j}}{h}+\frac{v_{i, j}}{2}\right) c_{i-1, j+1}=m_{i, j} c_{i, j}+s_{i, j}-s_{i, j+1} .
\end{aligned}
$$

To find values $c_{i, j+1}$ on $(j+1)$ th layer in (13), we first need to find value of function $s_{i, j+1}$ on this layer in (12).

Initial and boundary conditions $(7),(9),(8)$ for functions $c(x, t), s(x, t)$ can be rewritten as $c_{i, 0}=c_{0}(i h), s_{i, 0}=s_{0}(i h), c_{0, j}=c_{b 0}(j q), c_{k, j}=c_{b 1}(j q), i=\overline{0, k}, j=\overline{0, p}$.

\section{SWEEP METHOD FOR DIFFERENCE EQUATIONS}

We introduce the notations: $c_{i, j+1}=y_{i}, c_{i+1, j+1}=y_{i+1}, c_{i-1, j+1}=y_{i-1}, m_{i, j} c_{i, j}+s_{i, j}-s_{i, j+1}=$ $\varphi_{i},-\frac{q}{h}\left(\frac{D_{i, j}}{h}+\frac{v_{i, j}}{2}\right)=a_{i}, m_{i, j}+\frac{2 D_{i, j} q}{h^{2}}=b_{i},-\frac{q}{h}\left(\frac{D_{i, j}}{h}-\frac{v_{i, j}}{2}\right)=d_{i}$. Then (13) can be written as a system $(k+1)$ equations:

$$
\begin{aligned}
& y_{0}+0 \cdot y_{1}=c_{0, j+1}, \\
& a_{i} y_{i-1}+b_{i} y_{i}+d_{i} y_{i+1}=\varphi_{i}, \quad i=\overline{1, k-1}, \\
& 0 \cdot y_{k-1}+y_{k}=c_{k, j+1},
\end{aligned}
$$

i.e., for each $j=\overline{0, p-1}$ this is a linear system with three-diagonal matrix w.r.t. variables $y_{0}, \ldots, y_{k}$. The solution exists, is unique and it can be found by a sweep method.

\section{NUMERICAL EXPERIMENTS}

Once the difference scheme had been constructed, we made numerical calculations. As an illustration, we provide the results for the following data: $m=0.5$ is the media porosity, $D=1$ is the diffusion coefficient, $v=0$ is the filtration rate, $\Gamma=0.2$ is the Henry constant, $c_{0}(x)=\sin \pi x, x \in(0,1), s_{0}(x)=0, x \in(0,1), c_{b 0}(t)=0, c_{b 1}(t)=0, t \in(0, T), T=0.15$, $h=0.1$ is the step in $x, q=0.005$ is the step in $t, \tau_{1}=0.1, \tau_{2}=0.01$ are two values of character relaxation time.

The graphic visualization of calculations for $c(x, t)$ as $\tau=0.1$ is given in Figure 1, while for $s(x, t)$ is is given in Figure 2 .

Graphic visualization of the calculations for $c(x, t)$ as $\tau=0.01$ is given in Figure 3, and Figure shows the same for $s(x, t)$. 


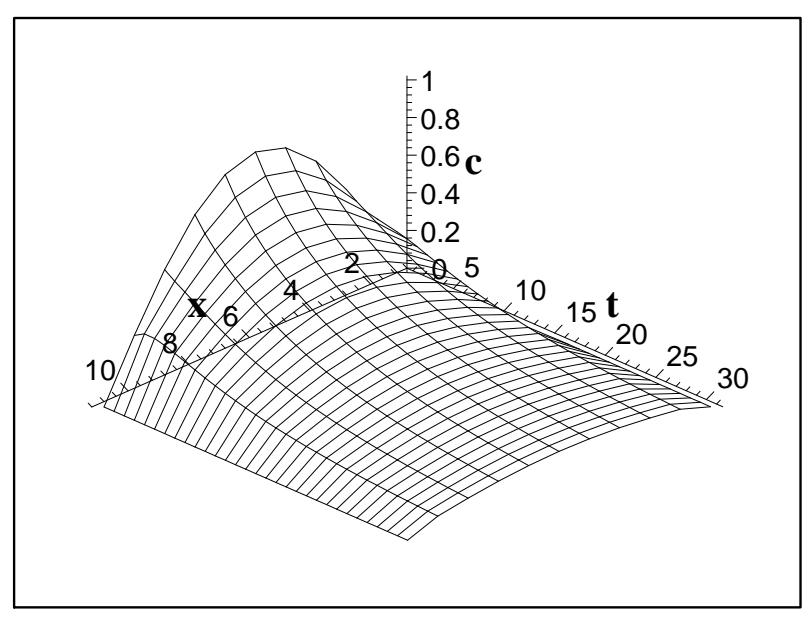

FiguRE 1.

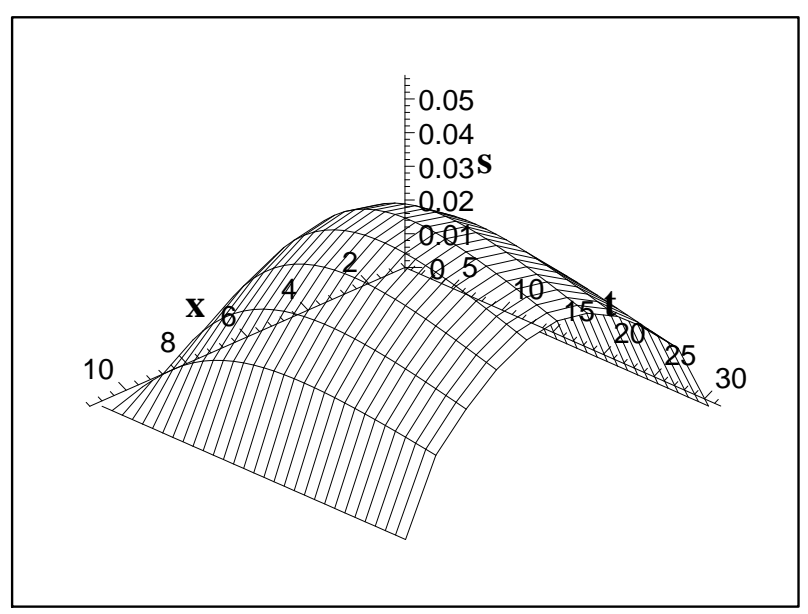

FigURE 2.

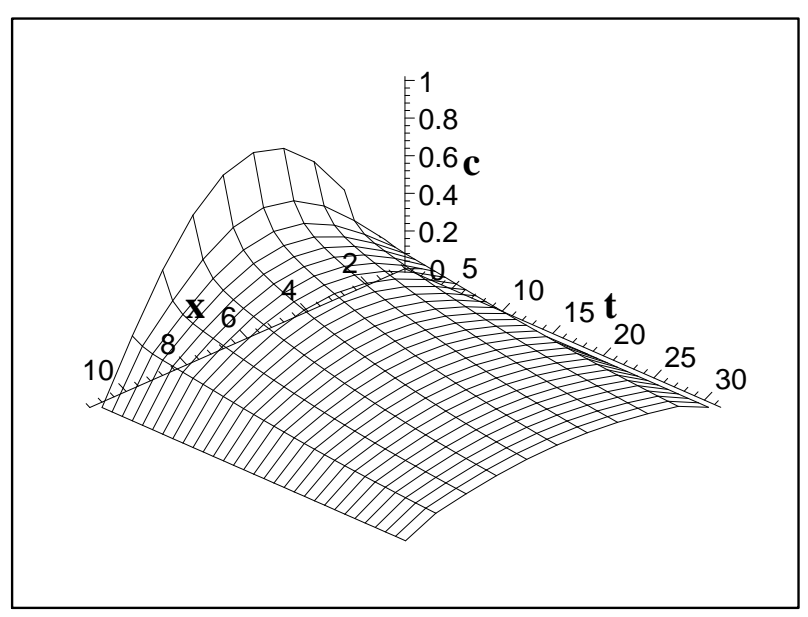

FiguRE 3.

\section{CONCLUSiON}

Basing on the numerical results and their visualization as the graphs, we can make the following conclusions. As relaxation time $\tau$ decreases, the solution to the non-equilibrium 


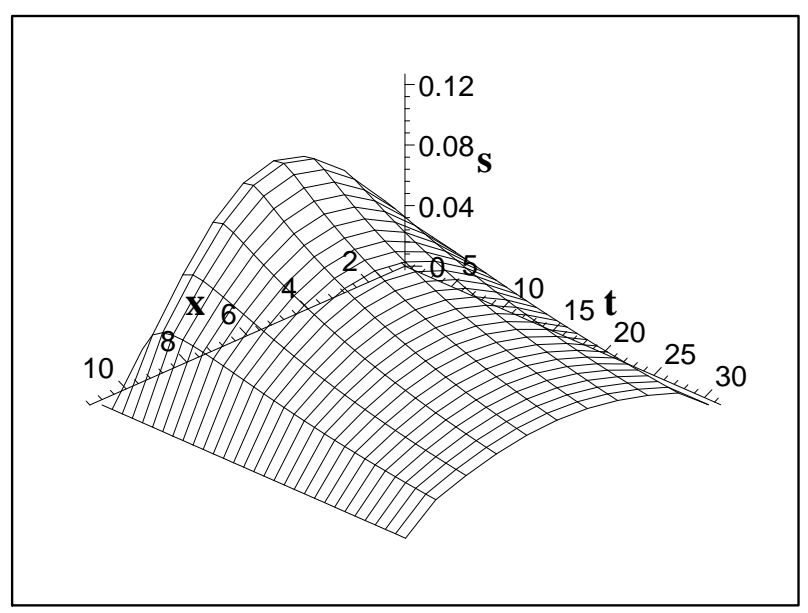

FiguRE 4.

problem tends to the solution of the equilibrium problem as the time grows, i.e., $s \rightarrow \Gamma c$. The obtained results allow us to confirm the predictions of theoretical studies and analytic results.

\section{BIBLIOGRAPHY}

1. L. Lapidus, W.R. Amundson. Mathematics of adsorption in beds. VI. The effect of longitudinal diffusion in ion exchange and chromatographic columns // J. Phys. Chem. 56:8, 984-988 (1952).

2. K.H. Coats, B.D. Smith. Dead and pore volume and dispersion in porous media // Soc. Petrol. Eng. J. 4:1, 73-84 (1964).

3. P.Ya. Polubarinova-Kochina (ed.) Development of studies on filtration theory in USSR. Nauka, Moscow (1969). (in Russian).

4. I.A. Kaliev, G.S. Sabitova. On a problem of nonequilibrium sorption // Sibir. Zhurn. Industr. Matem. 6:1, 35-39 (2003). (in Russian).

Ibragim Adietovich Kaliev,

Sterlitamak branch

of Bashkir State University,

Lenin av. 47,

453103, Sterlitamak, Russia

E-mail: kalievia@mail.ru

Saltanbek Talapedenovich Mukhambetzhanov,

Al-Farabi Kazakh National University,

al-Farabi av. 71,

050040, Almaty, Kazakhstan

E-mail: mukhambetzhanov_@mail.ru

Gul'nara Sagyndykovna Sabitova,

Sterlitamak branch

of Bashkir State University,

Lenin av. 47,

453103, Sterlitamak, Russia

E-mail: sabitovags@mail.ru 